\title{
Properties and Suitability of East Aqaba Area Feldspar for Glass Industries in Jordan
}

\author{
Saleh Shakhatreh ${ }^{1}$ \\ ${ }^{1}$ Department of Basic Sciences, AL-Huson University College, AL-Balqa' Applied University, Irbid, Jordan \\ Correspondence: Saleh Shakhatreh, Department of Basic Sciences, AL-Huson University College, AL-Balqa' \\ Applied University, P. O. Box 50, AL-Huson 21510, Jordan. Email: shakhatreh_s@yahoo.com
}

doi:10.5539/jmsr.v4n2p22

URL: http://dx.doi.org/10.5539/jmsr.v4n2p22

\begin{abstract}
In this study, a pegmatite ore with a $\mathrm{Na}_{2} \mathrm{O} / \mathrm{K}_{2} \mathrm{O}(5.54-4.29)$ ratio of 1.29 was studied in a Denver flotation cell in which both at natural and acidic $\mathrm{pH}$ using $\mathrm{HF}$ and $\mathrm{H}_{2} \mathrm{SO}_{4}$. Because of the perthitic structure of the ore no significant separation is observed at natural $\mathrm{pH}$. Interestingly, a selective separation was achieved at low $\mathrm{pH}$ using $\mathrm{HF}$ and $\mathrm{H}_{2} \mathrm{SO}_{4}\left(\mathrm{pH} 2-3\right.$ ). a pegmatite ore composed of $5.53 \% \mathrm{Na}_{2} \mathrm{O}$ and $4.29 \% \mathrm{~K}_{2} \mathrm{O}$ is upgraded to $10.51 \%$ $\mathrm{Na}_{2} \mathrm{O}$ and $6.59 .02 \% \mathrm{~K}_{2} \mathrm{O}$ with a $\mathrm{Na}_{2} \mathrm{O} / \mathrm{K}_{2} \mathrm{O}=1.62$ in $\mathrm{HF}$ medium and to 2.55 in $\mathrm{H}_{2} \mathrm{SO}_{4}$ medium. It is shown that products recovered in various stages are also considered as commercially significant; especially quartz which is recovered in the tailings in HF medium is suitable for glass industry.
\end{abstract}

Keywords: flotation, dry screen analysis, crushing, screening, heavy liquid separation

\section{Introduction}

Feldspar is the most common rock-forming mineral occurs in igneous, metamorphic, sedimentary, and numerous granitic rocks and thus can be found throughout Aqaba at south of Jordan. Feldspar weathers to kaolin which is the main clay mineral used mainly in the manufacture of ceramics, glass, and fine pottery. Feldspar minerals subdivided into plagioclase feldspars, and potassium feldspars. Plagioclase feldspars are sodium, calcium, aluminum, and silicates. The plagioclase feldspar is albite (moonstone) (sodium rich), anorthite (calcium rich), andesine, Oligoclase, and labradorite. Two properties makes feldspar useful for downstream industries, which is alkali and alumina contents, therefore we can distinguish their families as Feldspathic, pegmatite and Feldspar. Further distinction can be made between sodium potassium and mixed Feldspars depending on the type of alkali they contain. Feldspar plays an important role as fluxing agent in ceramics and glass application and also used as functional fillers in the paint plastic, rubber and adhesive industries.

It is found that the area of Aqaba city is the best for my study, because large amount of alkali feldspar granite raw materials on the surface containing about $71 \%$ potassium, $23 \%$ sodium and the proportion of quarts in purities mostly 5.5 mere bat of iron. Also you find the effect of the tectonic movements on the area cracked and shattered a large part of the rocks in different ways affecting mostly the middle part of the region. Moreover the way of reaching the area is very easy either by roads or valleys.

Generally, feldspar is used in the manufacture of glass products $(70 \%)$, ceramics and other products (30\%) (Potter1996). Feldspar is an important ingredient in the manufacture of glass. The raw material for glass consists of silica sand, soda ash (sodium carbonate) and limestone (calcium carbonate). Feldspar adds certain qualities to the process. Alumina provides hardness, workability, strength, and makes glass more resistant to chemicals. $\mathrm{Na}_{2} \mathrm{O}$ and $\mathrm{K}_{2} \mathrm{O}$ from feldspar are fluxes that reduce the melting temperature, so decrease the energy used and decrease the amount of soda ash needed (Kauffman \& Van Dyk, 1994; Bourne, 1994). About 110 pounds of feldspar are used to produce one ton of container glass (soda bottles, e.g.), and 100 pounds are required to produce one ton of flat glass (Alex Glover, 1999, pers. com). The alkali content in feldspar is an important ingredient in the manufacture of glass, because it acts as a fluxing agent, reducing the melting temperature of quartz and helping to control the viscosity of glass, thus reducing production costs. While alumina acts as a stabilizer and improves the finished product by increasing resistance to impact, bending, thermal shock, increases viscosity during glass formation, and inhibits devitrification. 


\section{Processes of Collecting Samples}

For focusing purposes three samples were collected fully representative from three parts of the region of the study at Aqaba south of Jordan as follows:

Composite sample no. 1 represents central part, where the sample collected from 69 different sites a round., where the amount of each sample was fixed by $10 \mathrm{~kg}$. Composite samples have deferent sizes of rocks; they were crushed to obtain soft and smooth powder for chemical analysis.

\section{Laboratory Chemical Charactrectes}

Chemical analysis were conducted by calibration size process after resolution of one gram of the composite sample powder in $10 \mathrm{ml}$ of Chloride acid and water then heated until boiling for ten minutes; all the compounds dissolved except silicon dioxide. The sediment of the filtered composite sample powder by useless ash paper washed with distilled water several times and the resulting sludge incinerated at 1000 degrees Celsius for three hours in order to determine the proportion of silicon dioxide in the composite samples. The output of the filtered composite sample were taken and put in a $100 \mathrm{ml}$ beaker and later used for chemical analysis to determine $\mathrm{Fe}_{2} \mathrm{O}_{3}$ and $\mathrm{Al}_{2} \mathrm{O}_{3}$ in sedimentation by Sodium Hydroxide Solution of 0.1 concentrations in presence of methyl red detector until the color change to yellow. The resulting sludge faltered by useless ash paper washed then calcining the sediment as in the case of determining Silicon. For iron proportion I toke a portion of the sediment output filtered by useless ash paper and washed by distilled water, then resolve the resulting oxides solution of acid with concentrated chlorine and heated to calibrate iron using a solution of EDTA in presence of salicylic acid detector, and the difference identified aluminum. Determining the $\mathrm{MgO}$ and $\mathrm{CaO}$ by calibrate a solution of EDTA in presence of Erochrom black $\mathrm{T}$ detector. For determining $\mathrm{K}_{2} \mathrm{O}$ and $\mathrm{Na}_{2} \mathrm{O}$ we used flame photometer process.

Table 1. Analysis of the Composite Samples in laboratory

\begin{tabular}{llllllllllll}
\hline Sample no. & $\mathrm{LOI}$ & $\mathrm{SiO}_{2}$ & $\mathrm{CaO}$ & $\mathrm{MgO}$ & $\mathrm{Fe}_{2} \mathrm{O}_{3}$ & $\mathrm{Al}_{2} \mathrm{O}_{3}$ & $\mathrm{TiO} 2$ & $\mathrm{Na}_{2} \mathrm{O}$ & $\mathrm{K}_{2} \mathrm{O}$ & $\mathrm{Mn}$ & $\mathrm{Na}_{2} \mathrm{OK}_{2} \mathrm{O}$ \\
\hline Sample 1 & 1.04 & 72.46 & 2.46 & 0.25 & 1.32 & 13.98 & 0.88 & 5.53 & 4.29 & 0.02 & 9.82 \\
Sample 2 & 1.31 & 74.72 & 2.04 & 0.20 & 0.37 & 13.63 & 0.92 & 5.34 & 4.34 & 0.03 & 9.68 \\
Sample 3 & 1.78 & 72.33 & 2.22 & 0.42 & 0.81 & 12.90 & 0.32 & 5.54 & 3.05 & 0.03 & 8.59 \\
\hline
\end{tabular}

To audit the results obtained in the laboratory through chemical analysis we identified the proportion of acids that the composite samples constitute by XRF ray on XRF device, and the results was almost compliance as we see in tables1\&2. The normative classification for the three composite samples represented in table 4.

Table 2. Analysis of the Composite Samples by X.R.F

\begin{tabular}{llllllllllll}
\hline Sample no. & $\mathrm{LOI}$ & $\mathrm{SiO}_{2}$ & $\mathrm{CaO}$ & $\mathrm{MgO}$ & $\mathrm{Fe}_{2} \mathrm{O}_{3}$ & $\mathrm{Al}_{2} \mathrm{O}_{3}$ & $\mathrm{TiO} 2$ & $\mathrm{Na}_{2} \mathrm{O}$ & $\mathrm{K}_{2} \mathrm{O}$ & $\mathrm{Mn}$ & $\mathrm{Na}_{2} \mathrm{OK}_{2} \mathrm{O}$ \\
\hline Sample1 & 1.01 & 72.20 & 2.22 & 0.23 & 1.31 & 13.91 & 0.87 & 5.58 & 4.28 & 0.03 & 9.86 \\
Sample2 & 1.32 & 74.83 & 2.28 & 0.19 & 0.40 & 13.71 & 0.91 & 5.39 & 4.42 & 0.05 & 9.81 \\
Sample3 & 1.77 & 72.31 & 2.28 & 0.53 & 0.84 & 12.95 & 0.38 & $5 . .57$ & 3.01 & 0.03 & 8.58 \\
\hline
\end{tabular}

Table 3. Chemical Composite

\begin{tabular}{llll}
\hline Minirals & & & \\
\hline Quartz & 23.12 & 25.08 & 21.91 \\
Orthoclase KAlSi3O8 & 23.05 & 25.02 & 23.91 \\
Albite Plagioclase NaAlSi3O8 & 45.53 & 41.82 & 48.21 \\
Anorthite Ca Al Si2O3 & 2.05 & 1.89 & 0.56 \\
Mafic & 5.95 & 6.19 & 6.02 \\
Orthoclase+ Albite +Anorthite & 70.63 & 68.73 & 72.68 \\
\hline
\end{tabular}


Table 4. Normative Classification of Composite Samples

\begin{tabular}{lllll}
\hline \multicolumn{2}{l}{ Chemical composition } & Samples 1 & Samples 2 & Samples 3 \\
\hline Potassium Oxides & $\mathrm{k} 2 \mathrm{O}$ & 25.37 & 26.23 & 20.41 \\
Sodium Oxides & $\mathrm{Na} 2 \mathrm{O}$ & 46.73 & 45.13 & 46.81 \\
Calcium Oxides & $\mathrm{Ca} 2 \mathrm{O}$ & 0.63 & .0 .09 & 0.12 \\
Mafic Group Oxides & $\mathrm{Fe} 2 \mathrm{O} 3+\mathrm{TiO} 3+\mathrm{MgO}$ & 5.27 & 5.54 & 6.00 \\
Quartz & 22.00 & 23.01 & 30.07 \\
Total & 100.00 & 100.00 & 100.00 \\
Feldspar & 72.73 & 71.45 & 67.34 \\
\hline
\end{tabular}

\section{Chemical Aspect}

The results of chemical analysis of the composite samples showed that ore granite Alkali Feldspar is of medium Grade kind (Contain 8.5- 9.5 percent of the oxides of sodium and potassium). Therefore to meet the specifications required for the manufacture of white Transparent glass, and industries of high quality of ceramic tiles, we should reduce the amount of impurities to the minimum rate that required to get a fine product without impurities of iron compounds, magnesium compounds and titanium compounds, as well as alleviate the proportion of silica, and separate the pure feldspar into two types which is Sodium spar and Potassium spar.

\section{Mechanical Laboratory Process}

Table 5. dry screen analysis of the composite samples

\begin{tabular}{|c|c|c|c|c|c|c|c|c|c|c|}
\hline \multirow{2}{*}{$\begin{array}{l}\text { Size Fraction in } \\
\mathrm{mm}\end{array}$} & \multirow[t]{2}{*}{$\mathrm{Wt} \%$} & \multirow{2}{*}{$\begin{array}{l}\text { Cumulative } \\
\text { Wt. \% }\end{array}$} & \multicolumn{2}{|c|}{$\mathrm{Na} 2 \mathrm{O} \%$} & \multicolumn{2}{|c|}{$\mathrm{K} 2 \mathrm{O} \%$} & \multicolumn{2}{|c|}{$\mathrm{A} 12 \mathrm{O} 3 \%$} & \multicolumn{2}{|c|}{$\mathrm{SiO} 2 \%$} \\
\hline & & & Assay & Units & Assay & Units & Assay & Units & Assay & Units \\
\hline+2 & 6.53 & 6.53 & 4.21 & 27.49 & 4.61 & 30.10 & 13.64 & 89.07 & 71.60 & 467.55 \\
\hline$-2+1$ & 27.68 & 34.21 & 6.00 & 166.08 & 4.35 & 120.41 & 14.14 & 391.39 & 70.28 & 1945.35 \\
\hline$-1+1 / 2$ & 10.06 & 44.27 & 5.12 & 51.51 & 4.55 & 45.77 & 13.29 & 133.70 & 70.44 & 708.68 \\
\hline$-1 / 2+1 / 2$ & 10.82 & 55.09 & 5.28 & 57.13 & 4.41 & 51.61 & 13.57 & 146.83 & 70.90 & 767.25 \\
\hline$-6.35+4$ & 6.76 & 61.85 & 6.02 & 40.69 & 4.43 & 29.95 & 14.14 & 95.59 & 71.34 & 482.26 \\
\hline$-4+2$ & 8.11 & 69.96 & 6.25 & 50.69 & 4.53 & 36.74 & 14.19 & 115.05 & 70.49 & 571.67 \\
\hline$-2+1$ & 7.77 & 77.73 & 4.85 & 37.68 & 4.43 & 34.89 & 13.23 & 102.80 & 70.99 & 551.59 \\
\hline$-1+0.5$ & 7.58 & 85.31 & 5.35 & 40.55 & 4.71 & 35.70 & 14.78 & $112 . .03$ & 70.90 & 537.42 \\
\hline$-05+0.297$ & 4.29 & 89.80 & 5.68 & 24.37 & 4.10 & 17.59 & 13.75 & 30.48 & 70.39 & 301.97 \\
\hline$-297+210$ & 2.12 & 91.72 & 5.18 & 10.98 & 437 & 9.26 & 14.38 & 39 & 71.36 & 151.28 \\
\hline$--210+149$ & 2.80 & 94.52 & 5.25 & 14.70 & 4.36 & 1221 & 14.10 & 48 & 70.92 & 198.58 \\
\hline$-149+74$ & 2.28 & 96.80 & 5.40 & 12.31 & 4.24 & 9.67 & 14.40 & 32.83 & 72.86 & 163.84 \\
\hline$-74+53$ & 0.58 & 97.38 & 5.18 & 3.00 & $4 . .13$ & 2.39 & 14.50 & 8.41 & 70.61 & 40.95 \\
\hline-53 & 2.62 & 100.00 & 5.31 & 13.21 & 493. & 10.30 & 14.68 & 38.46 & 70.62 & 185.02 \\
\hline calculated & 100.00 & & 5.51 & & 4.43 & & 13.95 & & 70.73 & \\
\hline Chemical Analysis & & 5.53 & & 4.29 & & 13.98 & & 71.46 & & \\
\hline
\end{tabular}

The ore feldspar granite of the composite samples for Laboratory tests were mechanically processed as follows:

Firstly dry screen analysis processed by drying the composite samples then screening the composite samples for 20 Minutes with screening machine (RO-Tap) Tyler Testing Sieve Shaker of type (Tyler Sieve System). Secondly dry screen analysis of the composite samples after crushing plus minus 2MM Table 5.

Note that the mechanical dry analysis had no impact on the metals as oxides of sodium and potassium silica oxide, since these metals distributed by different proportions to the all different sizes. Chemical analysis of different size fraction of the dry screen of the first composite sample is represented in Table 6. 
Table6. Chemical analysis of different size fraction

\begin{tabular}{lcccccccc}
\hline \multirow{2}{*}{ size Fraction in $\mathrm{mm}$} & \multicolumn{2}{c}{ Composite Sample 1 } & \multicolumn{2}{c}{ Composite Sample 2 } & \multicolumn{2}{c}{ Composite Sample 3 } & \multicolumn{2}{c}{ Composite Samples 1+2+3 Average by calculation } \\
\cline { 2 - 8 } & Wt.\% & Cumulate Wt.\%. & Wt.\% & Cumulate Wt.\%. & .Wt\% & Cumulate Wt.\%. & Wt\%. & Cumulate Wt.\% \\
\hline$-2+1$ & 26.53 & 26.53 & 28.21 & 28.21 & 27.61 & 27.61 & 27.56 & 27.56 \\
$-1+0.50$ & 27.58 & 54.11 & 30.57 & 58.78 & 28.07 & 55.68 & 28.89 & 56.45 \\
$-0.50+0.297$ & 13.62 & 67.73 & 12.87 & 71.65 & 13.69 & 69.37 & 13.37 & 69.82 \\
$-0.297+0.210$ & 7.28 & 75.01 & 7.10 & 78.75 & 7.38 & 76.75 & 7.25 & 77.07 \\
$-210+194$ & 6.95 & 81.96 & 5.37 & 84.09 & 6.53 & 83.28 & 6.19 & 83.26 \\
$-149+105$ & 4.59 & 86.55 & 4.10 & 88.19 & 4.20 & 87.48 & 4.26 & 87.52 \\
$-105+74$ & 2.60 & 89.15 & 2.20 & 90.39 & 2.86 & 90.34 & 2.54 & 30.06 \\
$-74+53$ & 4.41 & 93.56 & 4.30 & 94.69 & 3.96 & 94.30 & 4.20 & 94.26 \\
-53 & 6.44 & 100.00 & 5.31 & 100.00 & 5.70 & 100.00 & 5.74 & 100.00 \\
total & 100.00 & & 100.00 & & 100.70 & & 100.00 & \\
\hline
\end{tabular}

\section{Crushing and Screening}

Crushing and screening of the composite samples done in two phases (Figure 1):

Phase 1; Crushing the crude to pass from a sieve of size $25.4 \mathrm{~mm}$, in which the primary crushing used Jaw crushing machine.

Phase 2; Crushing the crude to pass from a sieve of size $2 \mathrm{~mm}$, in which the secondary crushing used a canonical crushing machine.

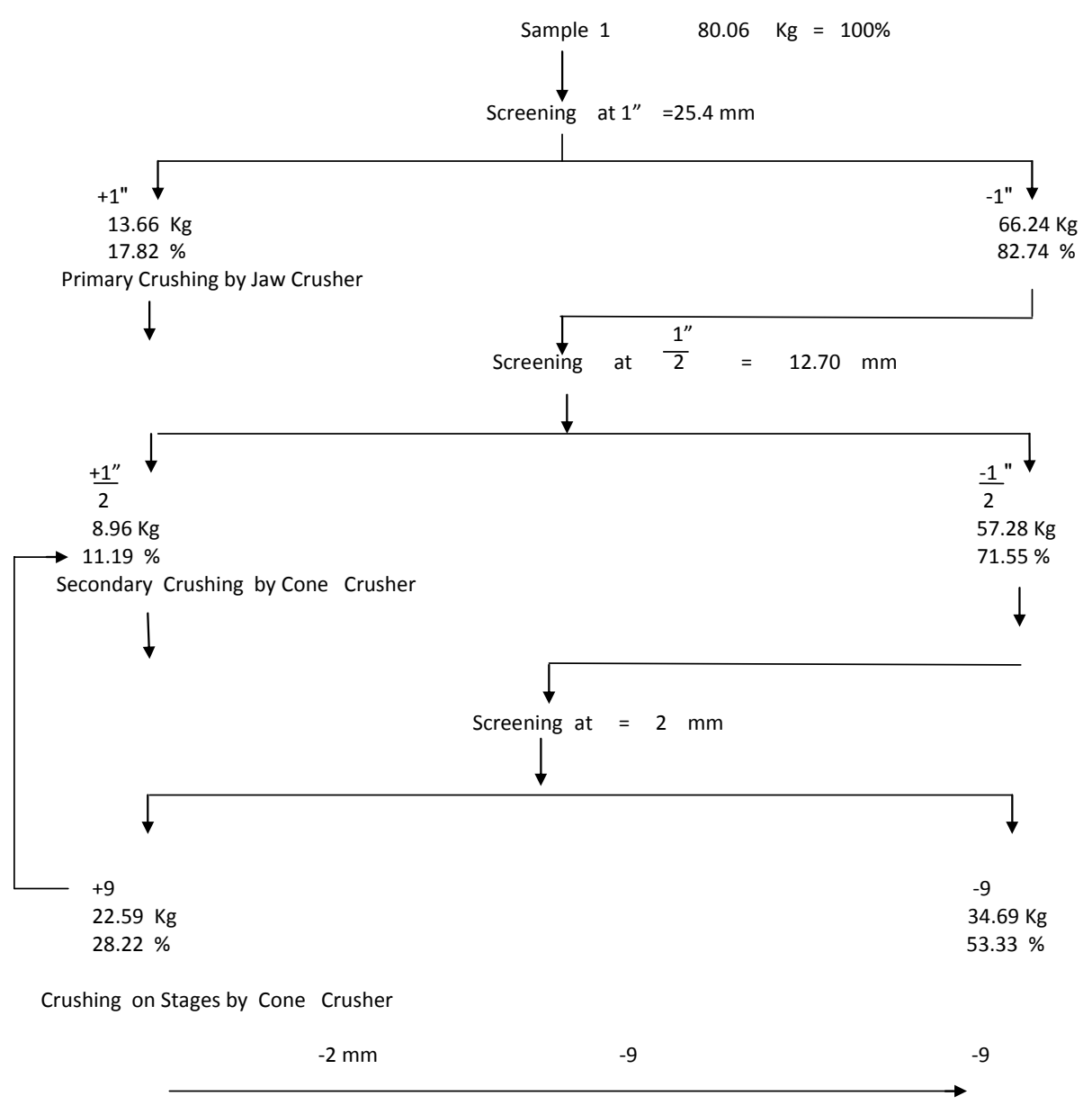

Figure 1. Process of crushing and screening of the composite samples 


\section{Separation Using Heavy Liquid}

Consecutive tests conducted on the raw feldspar using heavy liquid Bromoform to illustrate the concentrations of various density we get:

1-Best density quality of the liquid of heavy Bromoform to separate 90-95 percent of a collection of black metals was $2.594 \mathrm{~g} / \mathrm{ml}$. Table7.

2-Best density quality of the liquid of heavy Bromoform to separate black metals plus silica was $2.61 \mathrm{~g} / \mathrm{ml}$. Tables $8 \& 9$.

Table 7. Degree of Liberation

\begin{tabular}{|c|c|c|c|c|c|c|c|}
\hline \multirow{3}{*}{\multicolumn{2}{|c|}{ Size Mesh and Micron }} & \multicolumn{4}{|c|}{ Percentage of Liberated Minerals } & \multirow{3}{*}{ Contaminated grains } & \multirow{3}{*}{ Total $\%$} \\
\hline & & \multicolumn{2}{|c|}{ Feldspar } & \multirow{2}{*}{ Quartz } & \multirow{2}{*}{ Mafic Biotite } & & \\
\hline & & Orthoclase & Plagioclase & & & & \\
\hline+32 Mesh & $500 \mathrm{mic}$ & 42.2 & 18.0 & 23.7 & 3.2 & 12.50 & 99.30 \\
\hline+48 Mesh & $297 \mathrm{mic}$ & 47.0 & 13.3 & 23.6 & 8.5 & 7.00 & 99.40 \\
\hline+65 Mesh & $210 \mathrm{mic}$ & 45.8 & 17.8 & 26.4 & 3.9 & 6.10 & 100.00 \\
\hline+100 Mesh & 149mic & 42.3 & 23.0 & 20.3 & 4.0 & 10.20 & 99.80 \\
\hline +150 Mesh & $105 \mathrm{mic}$ & 41.5 & 19.0 & 24.5 & 5.5 & 9.00 & 99.50 \\
\hline$+200 \mathrm{Mesh}$ & $74 \mathrm{mic}$ & 36.6 & 19.0 & 24.3 & 5.5 & 12.20 & 97.60 \\
\hline-200 & & 34.4 & 13.4 & 25.1 & 4.9 & 21.10 & 98.90 \\
\hline
\end{tabular}

Table 8. Heavy liquid separation1

\begin{tabular}{lllllllllllll}
\hline Products & $\mathrm{wt} \%$ & $\mathrm{Na} 2 \mathrm{O} \%$ & \multicolumn{2}{c}{$\mathrm{K} 2 \mathrm{O} \%$} & \multicolumn{2}{c}{$\mathrm{A} 2 \mathrm{O} 3 \%$} & \multicolumn{2}{c}{$\mathrm{SiO} \%$} & \multicolumn{3}{c}{$\mathrm{Fe} 2 \mathrm{O} \%$} \\
\hline \multirow{3}{*}{ Float } & & Ass & Unit & Ass & Unit & Ass & Unit & Ass & Unit & Ass & Unit \\
Sink & 94.68 & 6.09 & 576.60 & 4.54 & 429.85 & 13.89 & 1315.11 & 73.51 & 6959.93 & 0.25 & 23.67 \\
Calcul Assay & 5.32 & 4.31 & 22.93 & 2.37 & 12.61 & 11.83 & 62.94 & 52.77 & 280.74 & 12.01 & 63.89 \\
& 100.00 & 5.99 & & 4.42 & & 13.78 & & 72.41 & & 0.88 & \\
\hline
\end{tabular}

Table 9. Heavy liquid separation 2

\begin{tabular}{|c|c|c|c|c|c|c|c|c|c|c|c|}
\hline \multirow[t]{2}{*}{ Products } & \multirow[t]{2}{*}{$\mathrm{wt} \%$} & \multicolumn{2}{|c|}{$\mathrm{Na} 2 \mathrm{O} \%$} & \multicolumn{2}{|c|}{ K2O\% } & \multicolumn{2}{|c|}{$\mathrm{A} 12 \mathrm{O} 3 \%$} & \multicolumn{2}{|c|}{$\mathrm{SiO} 2 \%$} & \multicolumn{2}{|c|}{$\mathrm{Fe} 2 \mathrm{O} 3 \%$} \\
\hline & & Assay & Recovery & Assay & Recovery & Assay & Recovery & Assay & Recovery & Assay & Recovery \\
\hline Float & 94.68 & 6.09 & 96.18 & 4.54 & 97.15 & 13.89 & 95.43 & 73.51 & 96.12 & 0.25 & 27.03 \\
\hline Sink & 5.32 & 4.31 & 3.88 & 2.37 & 2.85 & 11.83 & 4.57 & 52.77 & 3.88 & 12.01 & 72.97 \\
\hline $\begin{array}{l}\text { Calcul. } \\
\text { Assay }\end{array}$ & & & 100.00 & & 100.00 & & 100.00 & & 100.00 & & 100.00 \\
\hline
\end{tabular}

\section{Grinding and Screening}

For grinding the raw feldspar granite we used a rod laboratory machine of radius $25 \mathrm{~cm}$ and by percent of 60 solid materials as follows:

The raw materials inter for crashing and inter from screener of size $(2 \mathrm{~mm})$ on another screener of size 210 micro (65 meshes). The part above the screener to be used in the rod crushing machine, but the other part which interred the screener will be separated then interred through a screener of size 50 micro. Continuing this process for the raw materials we get

Micro $\quad \underline{\mathrm{Wt}}$.

$-210+50=75.96 \% \quad$ Feed for Flotation

$-50=24.04 \% \quad$ slime 
Table 10. The granite alkali feldspar is the most cruelty in rocks as in the following

\begin{tabular}{lcc}
\hline Minirals & Degree of Cruelty & Percentage $\%$ \\
\hline Silica & 7 & $23 \%$ \\
Sodium and potassium Oxides & 6 & $71 \%$ \\
Fe 2O3 & 6 & $6 \%$
\end{tabular}

The crashing of granite feldspars is very hard and costly; therefore we should crash it in three steps instead of two. Also it is better to crash the granite alkali feldspar when it is wet to avoid the problems in a dry way. After the process of grinding and screening we made the concentration of raw feldspar by multi flotation.

\section{Feldspar Differentiations Flotation}

Before any process of flotation we have to do scrubbing (washing), agitation and conditioning. Concentration of raw feldspar by multi-flotation done by three stages:

Table 11. Stage one; Flotation of iron compounds and the Biotite (Mica)

\begin{tabular}{|c|c|c|c|c|c|c|c|}
\hline \multicolumn{2}{|c|}{ Products } & $\begin{array}{l}\text { Feldspar CI. } \\
\text { Conc }\end{array}$ & $\begin{array}{l}\text { Feld. Rougher } \\
\text { Conc. }\end{array}$ & Feld.Tailing & $\begin{array}{l}\text { Mica+Iron } \\
\text { Float }\end{array}$ & Slime & Total \\
\hline \multirow[t]{2}{*}{$\mathrm{wt} \%$} & & 30.83 & 18.82 & 20.92 & 5.39 & 24.04 & \\
\hline & Assay & 62.06 & 66.72 & 82.86 & 52.77 & 63.42 & 67.11 \\
\hline \multirow[t]{3}{*}{$\mathrm{SiO} 2 \%$} & unite & 1913.31 & 1255.67 & 1733.43 & 1733.43 & 1524.62 & \\
\hline & recovery & 28.50 & 18.70 & 25.80 & 4.20 & 22.80 & \\
\hline & Assay & 0.24 & 0.02 & 0.08 & 12.01 & 4.30 & 1.78 \\
\hline \multirow[t]{3}{*}{$\mathrm{Fe} 2 \mathrm{O} 3 \%$} & unite & 7.40 & 0.38 & 1.67 & 64.73 & 103.85 & \\
\hline & recovery & 4.16 & 0.21 & 0.94 & 36.36 & 58.33 & \\
\hline & assay & 0.28 & 0.06 & 0.05 & 4.82 & 1.72 & 0.78 \\
\hline \multirow[t]{3}{*}{$\mathrm{MgO} \%$} & unite & 8.63 & 1.13 & 1.05 & 25.98 & 41.35 & \\
\hline & recovery & 11.00 & 1.40 & 1.30 & 33.30 & 50.00 & \\
\hline & assay & 1.16 & 0.87 & 0.77 & 6.36 & 2.47 & 1.62 \\
\hline \multirow[t]{3}{*}{$\mathrm{CaO} \%$} & unite & 35.76 & 16.37 & 16.11 & 34.28 & 59.38 & \\
\hline & recovery & 22.07 & 10.10 & 9.94 & 21.16 & 36.65 & \\
\hline & assay & 10.65 & 8.89 & 2.84 & 4.31 & 3.67 & 6.66 \\
\hline \multirow[t]{3}{*}{$\mathrm{Na} 2 \mathrm{O} \%$} & unite & 328.34 & 167.31 & 59.41 & 23.23 & 88.23 & \\
\hline & recovery & 49.30 & 25.10 & 8.90 & 3.50 & 13.20 & \\
\hline & assay & 6.59 & 4.99 & 2.59 & 2.37 & 4.25 & 4.66 \\
\hline \multirow[t]{3}{*}{$\mathrm{K} 2 \mathrm{O} \%$} & unite & 203.17 & 93.91 & 54.18 & 12.77 & 102.17 & \\
\hline & recovery & 43.60 & 20.20 & 11.60 & 2.70 & 21.90 & \\
\hline & assay & 17.63 & 17.08 & 9.51 & 11.83 & 16.50 & 16.50 \\
\hline \multirow[t]{3}{*}{$\mathrm{A} 12 \mathrm{O} 3 \%$} & unite & 543.22 & 321.44 & 198.95 & 63.76 & 396.66 & \\
\hline & recovery & 35.64 & 21.09 & 13.05 & 4.185 & 26.02 & \\
\hline & assay & 0.54 & 0.79 & 0.50 & 4.76 & 2.31 & 1.23 \\
\hline \multirow[t]{2}{*}{ L.O.I\% } & unite & 16.65 & 14.87 & 10.46 & 25.66 & 55.53 & \\
\hline & recovery & 13.50 & 12.10 & 8.50 & 20.80 & 45.10 & \\
\hline
\end{tabular}


Table 12. Stage two; Flotation of feldspars

\begin{tabular}{llll}
\hline $\mathrm{wt} \%$ & Minerals & Assay\% & Recovery\% \\
\hline 49.65 & L.O.I & 0.66 & - \\
& $\mathrm{SiO} 2$ & 64.39 & 47.20 \\
& $\mathrm{~A} 2 \mathrm{O} 3$ & 18.35 & 61.00 \\
& $\mathrm{Na} 2 \mathrm{O}$ & 9.77 & 74.40 \\
& $\mathrm{~K} 2 \mathrm{O}$ & 5.79 & 63.80 \\
& $\mathrm{CaO}$ & 1.01 & - \\
& $\mathrm{MgO}$ & 0.17 & 12.40 \\
& $\mathrm{Fe} 2 \mathrm{O} 3$ & 0.13 & 4.37 \\
\hline
\end{tabular}

Table 13. Stage two; Flotation of feldspars

\begin{tabular}{cccccccc}
\hline \multicolumn{4}{c}{ Feldspar Clean Float } & \multicolumn{3}{c}{ Feldspar Rougher Float } \\
\hline WT\% & Minerals & Assay\% & Recovery\% & $\mathrm{Wt} \%$ & Minerals & Assay\% & Recovery\% \\
30.83 & L.O.I & 0.54 & 13.50 & 18.82 & L.O.I & 0.79 & 12.10 \\
& $\mathrm{SiO} 2$ & 62.06 & 28.50 & & $\mathrm{SiO} 2$ & 66.72 & 18.70 \\
& $\mathrm{~A} 2 \mathrm{O} 3$ & 17.62 & 38.25 & & $\mathrm{Al} 2 \mathrm{O} 3$ & 17.08 & 22.64 \\
$\mathrm{Na} 2 \mathrm{O}$ & 10.65 & 49.30 & & $\mathrm{Na} 2 \mathrm{O}$ & 8.89 & 25.10 \\
$\mathrm{~K} 2 \mathrm{O}$ & 6.39 & 43.60 & & $\mathrm{~K} 2 \mathrm{O}$ & 4.99 & 20.20 \\
$\mathrm{CaO}$ & 1.16 & - & & $\mathrm{CaO}$ & 0.87 & - \\
$\mathrm{MgO}$ & 0.28 & 11.00 & & $\mathrm{MgO}$ & 0.06 & 1.40 \\
$\mathrm{Fe} 2 \mathrm{O} 3$ & 0.24 & 4.16 & & $\mathrm{Fe} 2 \mathrm{O} 3$ & 0.02 & 0.21 \\
\hline
\end{tabular}

Table 14. Stage two; Flotation of feldspars

\begin{tabular}{cccc}
\hline $\mathrm{wt} \%$ & Minerals & Assay\% & Recovery $\%$ \\
\hline 43.19 & $\mathrm{~L} . \mathrm{O} . \mathrm{J}$ & 0.55 & 25.58 \\
& $\mathrm{SiO} 2$ & 60.20 & 37.34 \\
& $\mathrm{CaO}$ & 0.64 & 24.52 \\
& $\mathrm{MgO}$ & 0.29 & 19.65 \\
& $\mathrm{Fe} 2 \mathrm{O} 3$ & 0.47 & 11.73 \\
& $\mathrm{Al} 2 \mathrm{O} 3$ & 19.30 & 59.35 \\
& $\mathrm{Na} 2 \mathrm{O}$ & 11.08 & 73.86 \\
& $\mathrm{~K} 2 \mathrm{O}$ & 6.73 & 65.07 \\
\hline
\end{tabular}

Table 15. Stage three; the separation of feldspar for two types' sodium feldspar and potassium feldspar

\begin{tabular}{ccccccc}
\hline & \multicolumn{2}{c}{ Sodic feldspar } & \multicolumn{2}{c}{ Sodic feldespar } & \multicolumn{2}{c}{ Mixed feldspar } \\
\hline Minerals & Assay\% & $\mathrm{Wt} \%$ & Assay\% & $\mathrm{Wt} \%$ & Assay\% & $\mathrm{Wt} \%$ \\
$\mathrm{~L} . \mathrm{O} . J$ & 0.61 & & 0.35 & & 0.47 & \\
$\mathrm{SiO} 2$ & 64.98 & & 71.47 & & 70.67 & \\
$\mathrm{CaO}$ & 0.49 & & 0.71 & & 0.85 & \\
$\mathrm{MgO}$ & 0.06 & & 0.25 & & 0.33 & \\
$\mathrm{Fe} 2 \mathrm{O} 3$ & 0.30 & 16.61 & 0.48 & 23.90 & 0.93 & 13.19 \\
$\mathrm{Al} 2 \mathrm{O} 3$ & 14.67 & & 13.68 & & 14.47 & \\
$\mathrm{Na} 2 \mathrm{O}$ & 10.92 & & 8.49 & & 6.34 & \\
$\mathrm{~K} 2 \mathrm{O}$ & 7.31 & & 3.66 & & 4.67 & \\
Recovery\% & 25.94 & & 18.70 & \multicolumn{4}{c}{13.16} & Head Recovery $=57.80 \%$ \\
Head wt. $=53.69 \%$ & & \multicolumn{5}{c}{}
\end{tabular}




\section{Stages of Feldspar Flotation}

1-First stage: float iron compounds plus biotite (mica) used the following chemicals:

Sulphric Acid

$800-1000 \mathrm{gr} / \mathrm{ton}$

PH 3

Aeropromoter Collector

$600-800 \mathrm{gr} /$ ton

Dow Froth 1012

$20-50 \mathrm{gr} / \mathrm{ton}$

Kerosene

$60-80 \mathrm{gr} / \mathrm{ton}$

2-Second stage: flotation feldspar and quartz Thbit used the following chemicals:

Hydrofluoric Acid 72\%

$800-1000 \mathrm{gr} / \mathrm{ton}$

PH 3

Aranic T or Aminac T

$300-400 \mathrm{gr} / \mathrm{ton}$

Dow Froth 1012

$20-50 \mathrm{gr} / \mathrm{ton}$

Kerosene

$60-80 \mathrm{gr} / \mathrm{ton}$

3-Third stage: separation of feldspar product of the second phase into Sodium spar and potassium spar used the following chemicals:

Sodium Silcate

Aranic $\mathrm{T}$

Pine Oil

Kerosene
$500-600 \mathrm{gr} / \mathrm{ton}$

$250-300 \mathrm{gr} / \mathrm{ton}$

$60-80 \mathrm{gr} / \mathrm{ton}$

$60-80 \mathrm{gr} / \mathrm{ton}$

\section{Infrared Spectroscopic Analysis (IR)}

Infrared absorption spectra in the region of $400 \mathrm{~cm}^{-1}$ to $4000^{-1} \mathrm{~cm}^{-1}$ were recorded using $\mathrm{KBr}$ sample alkali feldspar. The samples were prepared as pellet method according to Russell and Fraser (1994). In the present work, a Bruker Vector spectrophotometer model FT-IR-22, Germany in the region of 4000-400 cm-1 used. Figures 2, 3, and 4 . Table 16 present the absorption spectrum in the infrared region for the collective samples and the absorption band at $3645 \mathrm{~cm}^{-1}$ is attributed to stretching vibrations of the $\mathrm{O}-\mathrm{H}$ group, while at $6454 \mathrm{~cm}^{-1}$ the presence of interlayer water and the amount of adsorbed water in clays is related to the deformation vibration of $\mathrm{H}-\mathrm{O}-\mathrm{H}$ group $1664 \mathrm{~cm}^{-1}$ where the band at $1042 \mathrm{~cm}^{-1}$ and $798 \mathrm{~cm}^{-1}$ are attributed to $\mathrm{Si}-\mathrm{O}$ stretching vibrations, the band at 770 $\mathrm{cm}^{-1}$ corresponds to the beidellite species, and the band at $526 \mathrm{~cm}^{-1}$ and $455 \mathrm{~cm}^{-1}$ correspond to deformation vibration of $\mathrm{Si}-\mathrm{O}-\mathrm{Al}$ and $\mathrm{Si}-\mathrm{O}-\mathrm{Si}$ respectively (volzone et al., 1988).

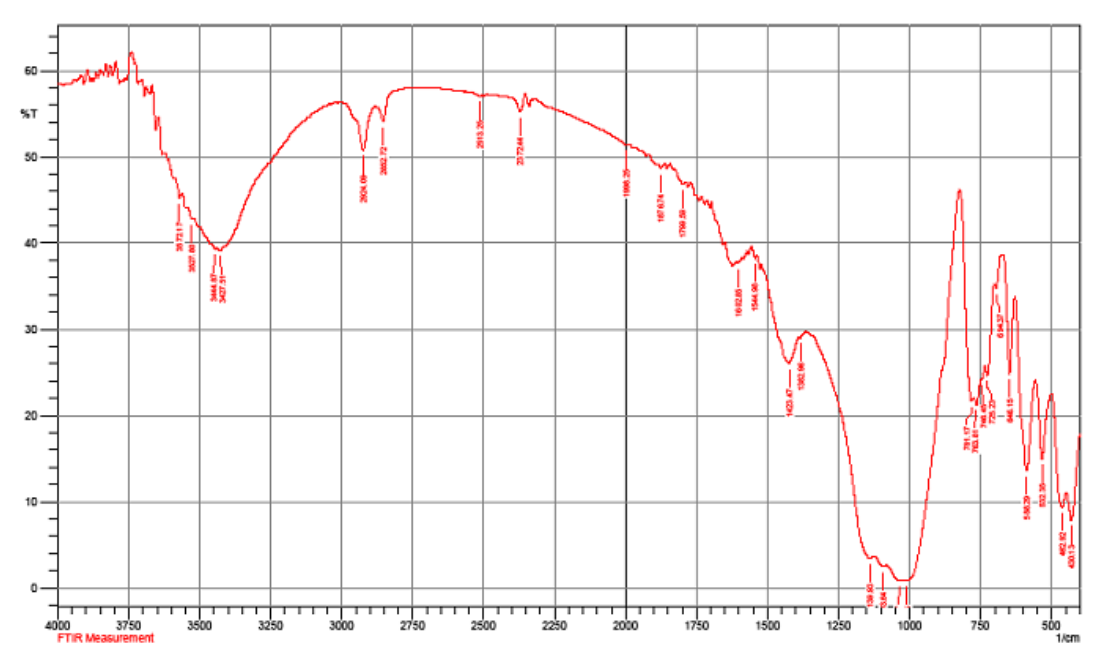

Figure 2. FTIR spectra of the examined sample 1 


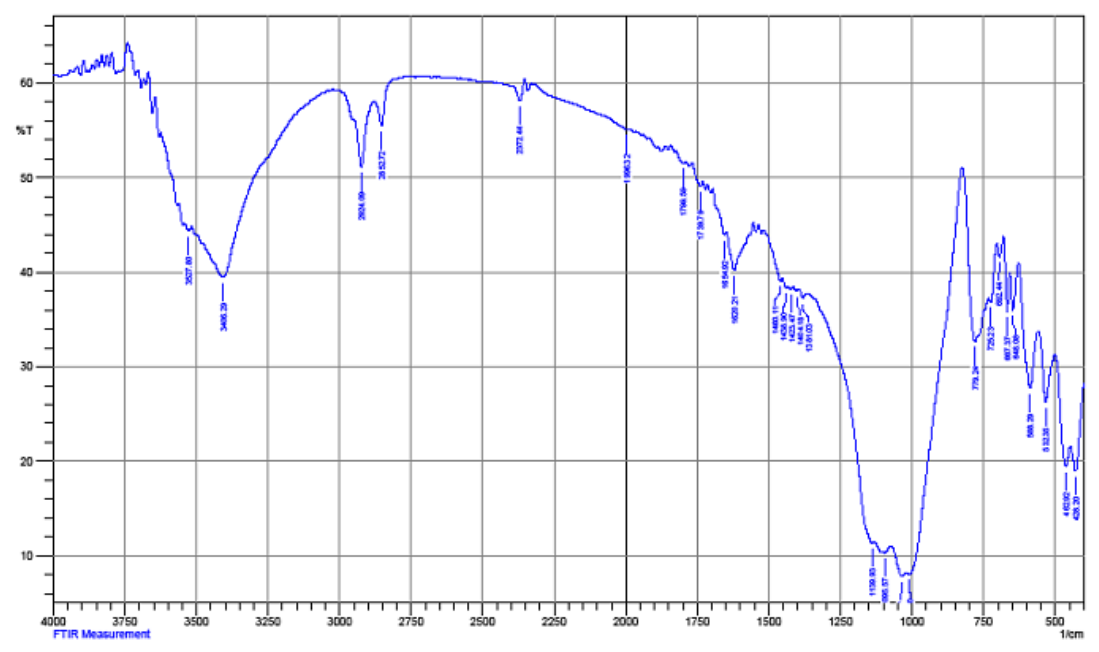

Figure 3. FTIR spectra of the examined sample 2

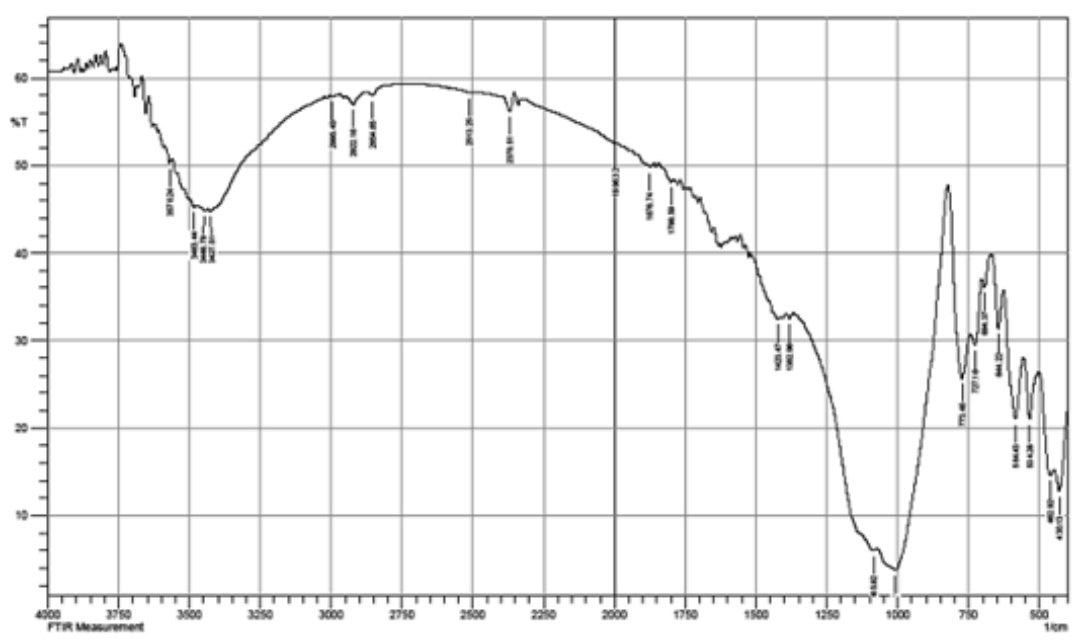

Figure 4. FTIR spectra of the examined sample 3

Table 16. Observed frequencies $(\mathrm{cm}-1)$ of the FTIR spectra for all samples

\begin{tabular}{|c|c|c|c|c|c|c|c|c|c|c|c|}
\hline \multirow{3}{*}{$\mathrm{O}-\mathrm{H}$} & \multicolumn{4}{|c|}{ Sample 1} & \multicolumn{3}{|c|}{ Sample 2} & \multicolumn{2}{|c|}{ Sample 3} & & \\
\hline & \multicolumn{4}{|c|}{ vibration bands } & \multicolumn{3}{|c|}{ vibration bands } & \multicolumn{2}{|c|}{ vibration bands } & \multirow[b]{2}{*}{3445} & \multirow[b]{2}{*}{3487} \\
\hline & 3572 & 3527 & 3444 & 3427 & 3527 & 3405 & & 3570 & 3464 & & \\
\hline & 2927 & 2852 & --- & --- & 2924 & 2852 & 2772 & 2995 & 2922 & 2854 & ---- \\
\hline \multirow{5}{*}{$\mathrm{H}-\mathrm{O}-\mathrm{H}$} & 2512 & 2372 & --- & --- & --- & --- & --- & 2513 & 2370 & --- & --- \\
\hline & 1999 & 1876 & --- & --- & 1806 & --- & --- & 1996 & 1876 & ---- & ---- \\
\hline & 1799 & 1602 & --- & --- & 1795 & 1739 & --- & 1790 & ----- & ---- & ---- \\
\hline & 1544 & 1423 & --- & --- & 1654 & 1620 & --- & 1362 & ----- & ---- & ---- \\
\hline & 1382 & 1382 & 1354 & --- & 1361 & ---- & ---- & 1423 & ----- & ---- & \\
\hline Si-0-Si(A1)-0 & ---- & ----- & --- & --- & 1059 & 1139 & ---- & 1165 & ----- & ---- & ---- \\
\hline $\mathrm{Si}-\mathrm{Si}$ & 753 & 745 & 725 & ---- & 779 & 725 & ---- & 725 & 727 & ---- & ---- \\
\hline $\mathrm{O}-\mathrm{Si}(\mathrm{AI})-0$ & 694 & 646 & ---- & ---- & 667 & 645 & ----- & ---- & ---- & ---- & ---- \\
\hline O-Si-O & 592 & --- & --- & --- & 566 & --- & ---- & 554 & --- & ---- & ----- \\
\hline $\mathrm{K}-\mathrm{O}, \mathrm{Na}-\mathrm{O}$ & 532 & --- & --- & --- & 532 & --- & --- & 534 & ---- & ---- & ---- \\
\hline $\mathrm{Si}-\mathrm{O}-\mathrm{Si}(\mathrm{Al})$ & --- & --- & --- & --- & 425 & --- & --- & 452 & 430 & ---- & --- \\
\hline
\end{tabular}




\section{XRD Determinations}

Samples were studied using X-ray technology X. R. D on a kind of Phillips, who has an elevator from the copper a wavelength-ray source $1.5401 \AA$, and the candidate of nickel in the area of $2 \theta: 10-70$, and has a software program for processing the data generated, depending on the values of $\theta$ taken from the curves and compared with standard curves of metals have been identified d representing the dimensions between levels crystalline, and linked to each of $\theta$ and d relationship Prague (Alberty \& Farrinction, 1978), and in doing so we got the Table 17, which shows the results of X-ray spectrum analysis.

Table 17. The results of X-ray analysis of the samples

\begin{tabular}{cccc}
\hline & Sample 1 & Sample 2 & Sample 3 \\
$2 \theta$ & $\mathrm{d}$ & $\mathrm{d}$ & $\mathrm{d}$ \\
\hline 08.90 & - & 9.92 & 10.2 \\
12.49 & - & 7.08 & - \\
13.89 & 6.36 & 6.38 & 6.38 \\
20.87 & 4.25 & 4.25 & 4.25 \\
21.06 & 4.21 & - & - \\
22.04 & 4.03 & 4.03 & 4.03 \\
23.04 & 3.85 & - & - \\
2355 & 3.77 & 3.77 & 3.77 \\
24.23 & 3.67 & 3.68 & 3.67 \\
25.63 & 3.47 & 3.47 & 3.48 \\
26.39 & 3.37 & - & - \\
26.63 & 3.34 & 3.34 & 3.34 \\
27.03 & 3.29 & - & 3.29 \\
27.52 & 3.23 & 3.23 & - \\
27.94 & 3.19 & 3.19 & 3.19 \\
28.16 & 3.16 & 3.16 & - \\
28.32 & 3.14 & - & - \\
29.88 & 2.99 & 2.98 & 2.98 \\
30.06 & 2.97 & - & - \\
30.47 & 2.93 & 2.93 & - \\
30.78 & 2.90 & - & - \\
31.26 & 2.86 & - & - \\
35.04 & 2.55 & - & - \\
36.54 & 2.45 & 2.46 & 2.46 \\
39.46 & 2.28 & 2.28 & - \\
40.26 & 2.24 & - & 2.24 \\
42.45 & 2.12 & 2.12 & 2.12 \\
45.79 & 1.97 & 1.98 & 1.98 \\
50.13 & 1.81 & 1.81 & 1.81 \\
51.14 & 1.78 & - & - \\
54.86 & 1.67 & 1.67 & 1.67 \\
59.94 & 1.54 & 1.54 & 1.54 \\
67.74 & 1.38 & 1.38 & 1.37 \\
\hline & & &
\end{tabular}


Fildsparia, we have got these results by comparing the absorption peaks shown in the table where the table shows the absorption peaks for these metals as follows:

\begin{tabular}{ll}
\hline Mineral & Range of $[\AA \AA]$ \\
\hline Feldspars. & $3.18-1.58$ \\
Albite Plagioclase NaAlSi3O8. & $3.19,4.03,3.2$ \\
Anorthite $\mathrm{Ca}$ Al2 Si2O3. & $3.20,3.18,4.04$ \\
Orthoclase KAlSi3O8. & $3.31,3.77,4.22$ \\
Quartz $\quad \mathrm{SiO} 2$ & $3.34,4.26,1.82$ \\
\hline
\end{tabular}

\section{Conclusion}

At the laboratory of Al- Huson University college Al- Balqa University we succeeded in producing Sodium feldspar and Potassium feldspar out of the three composite samples from Granite feldspar raw materials collected from different parts of the site of the study $6 \mathrm{~km}$ to the east of Aqaba city. The site of the study has a reserve of Millions of tons of the Granite feldspar raw Materials. The beneficiation analysis was successful in producing alkali feldspar, potassium alkali feldspar, sodium alkali feldspar which meets the proportion of the standard grade quality according to the Jordanian standard grade and the Industrial Minerals Handbook (Harben, 1999).

\section{Acknowledgements}

All analytical measurements were carried out in the XRF,I.R, XRD and chemical analysis laboratories of the. Royal Scientific Society and Natural Resources Authority. Their technical support is kindly acknowledged.

\section{References}

Alberty, R. A., \& Farrinction, D. (1978). Physical Chem. (5th ed., p. 598). John Wely and Sons.

Bourne, H. L. (1994), Glass raw materials. In D. D. Carr (Ed.), Industrial minerals and rocks (6th ed., pp. 543-550). Littleton, CO, Soc. for Mining, Metallurgy and Exploration, Inc.

Brady, G. S., \& Clauser, H. R. (1977). Materials Handbook (11th ed.). NY: McGraw-Hill Book Co.

Burger, J. (1990). Feldspar and nepheline syenite. Industrial minerals, 275, 21-33.

Harben, P. W. (1999). Silica and Quartz in The Industrial Minerals Handbook (3rd ed., pp. 183-190). Surrey, UK: Industrial Minerals Information Ltd.

Loughbrough, R. (1992). Italy's industrials. Industrial mineral, 301, 38-39.

Kauffman, R. A., \& Van Dyk, D. (1994). Feldspars. In D. D. Carr (Ed.), Industrial minerals and rocks (6th ed., pp. 473-481). Littleton, CO, Soc. for Mining, Metallurgy and Exploration, Inc.

Lesure, F. G. (1968). Mica deposits of the Blue Ridge in North Carolina: U.S. Geol. Sur. Prof. Paper 577.

Mineral Lab Inc. (2000). Methods and Results, October 13.

Murphy, T. D. (1960). Silica Sand and Pebble. In Industrial Minerals and Rocks (3rd ed., pp. 763-772). NY: The American Institute of Mining, Metallurgical, and Petroleum Engineers, Inc.

Peter W. H. (1999). The Industrial Minerals Handy Book (3rd ed.).

Potter, M.J., (1991). Feldspar, and Nepheline Syenite, and Aplite. Annual Report, US Dept .of I nterior, US Bureau of Mines.

Potter, M. J. (1996). Feldspar and Nepheline Syenite. Minerals Yearbook, US Dept. of Interior, US Geological Survey; (//minerals.er.usgs.gov/minerals).

Potter, M. J. (1997). Feldspar: Mineral Commodity Summaries. US Dept. of Interior, US Geological Survey; (http://minerals.er.usgs.gov/minerals).USM 1993, Short Course on : Industrial Processing.

Rao, K. H. anymantha \& Forssberg, K. S. E. (1993). Solution chemistry of mixed cationic/anionic collectors and Flotation of feldspar from quartz. Proc XVIII International Mineral Processing Congress Vol.4 Flotation II and miscellaneous (pp. 837-844). Australian Institute of Mining and Metallurgy.

Mensah-Biney, R., Carpenter, L. A., Allen, B. J., Miller, J. W., \& Reid, J. C. (2001). Glass sand potential or the Pinehurst Formation, Richmond County, North Carolina: Mineral identification and processing. $50^{\text {th }}$ Annual 
meeting and abstracts Raleigh, North Carolina, Southeastern Section. Geological Society of America, 33(2), A-2.

Volzone, C., Zalba, P. E., \& Pereira, E. ( 1988). Activation acid de esmectitas. II - Estudo mineralogical. Annals de la Association Química Argentina, 76, 57-68.

Wiener, L. S., Merchant, C. E., \& Tanner, J. T. Jr. (1990). High-silica resource potential of the Upper Chilhowee Quartzite, McDowell County, North Carolina: North Carolina Geological Survey. Information Circular, $26,22$.

Yachuan, L., Huangu, G., Jichuan, Q., \& Keren, Z. (1993) A new Flotation technique for Feldspar- quartz separation. Proc XVIII International Mineral Processing Congress Vol.4 Flotation II and miscellaneous (pp. 857-862). Australian Institute of Mining and Metallurgy.

\section{Copyrights}

Copyright for this article is retained by the author(s), with first publication rights granted to the journal.

This is an open-access article distributed under the terms and conditions of the Creative Commons Attribution license (http://creativecommons.org/licenses/by/3.0/). 